\title{
GENETIC LOADS AFFECTING FERTILITY IN NATURAL POPULATIONS OF DROSOPHILA PSEUDOOBSCURA ${ }^{1}$
}

\author{
DRAGOSLAV MARINKOVIC \\ The Rockefeller University, New York, N.Y. 10021 and Faculty of \\ Science, University of Belgrade, Yugoslavia
}

Received June 26, 1967

\begin{abstract}
A study of the genetic loads affecting fecundity of females of Drosophila pseudoobscura has recently been reported in this journal (Marinkovic 1967). It has been shown that females homozygous for second chromosomes derived from natural populations deposit on the average fewer eggs than do females carrying two second chromosomes derived from different wild progenitors. The present report extends the study to the egg-to-adult viability of the progenies of homozygous and heterozygous females, and thus permits estimation of the reduction of the fertility caused by the manifestation of these normally concealed genetic loads.
\end{abstract}

\section{MATERIALS AND METHODS}

Samples of Drosophila pseudoobscura were collected in Borrego Valley and Palm Canyon (California) by Mr. David Richmond, and near Tucson (Arizona) by Dr. William Heed, in November 1966. A total of 146 males (or sons of wild females) from these samples (92 from California and 54 from Arizona) were crossed individually to females from the laboratory strain with a dominant mutant marker (Bare) on the second chromosome. The analyzer stock was outcrossed (by Mr. Spassky) for several generations to flies of Arizona origin. Homozygotes and heterozygotes for wild-type second chromosomes were obtained by a standard technique described by Dobzranskx, Holz and Spassis y (1942), Marinkovic (1967) and others. Briefly, after backcrossing individual males from the $F_{1}$ generation to females from the Delta/Bare balanced strain, females and males heterozygous for the same wild chromosome and a chromosome with the mutant marker Bare were intercrossed (two pairs per bottle, three replications per each combination). In the following generation, the expected proportions of the wild-type homozygotes and Bare heterozygotes should be $33.3: 66.7$. Wild-type heterozygotes were obtained in a similar way, by crossing males and females with wild-type second chromosomes derived from different wild progenitors, in a series $1 \times 2,3 \times 4,5 \times 6$, etc. The experimental cultures were kept at $25^{\circ} \mathrm{C}$ in half-pint bottles with Spassky's nutrient medium.

The study of the fecundity was performed in the way described by MARunkovic (1967). From each of three replicate bottles three females (homo- or heterozygous for wild-second chromosomes) were taken less than 24 hours after emergence. Four unrelated males (from Delta/Bare strain) were added to each group of females and allowed to age together in the vials with a small amount of food. The egg laying capacity of the females was measured for six days, between the 6th and 12th day after emergence from the pupae. Vials were kept in an incubator at $25^{\circ} \mathrm{C}$ and $65-70 \%$ relative humidity. The egg laying capacity was measured in this way for 118 second chromosome homozygotes (complete lethals and semilethals with viability less than $10 \%$ were excluded), and for 87 heterozygous combinations (64 nonlethal and 23 lethal/nonlethal).

\footnotetext{
${ }^{1}$ The work reported in this article has been carried out under Contract No. AT-(30-1)-3096, U.S. Atomic Energy Commission.
}

Genetics 57 : 701-709 November 1967. 
From each of the three replicate cultures, a sample of 40 to 60 eggs was taken and transferred with a small piece of medium onto the surface of Spassky's medium, in a half-pint bottle. After 16 and 23 days, the numbers of emerged flies were counted, and the percentage survival of the progenies of homozygous or heterozygous mothers was estimated for all 118 second-chromosome homozygotes, and for 82 heterozygous combinations (62 nonlethal and 20 lethal/nonlethal). Some of the homozygous females whose eggs showed extremely low survival were dissected to observe abnormalities in their reproductive organs. Attempts have also been made to determine at which stages of embryogenesis development ceased (eggs older than 48 hours have been used). After dissolving the chorion in a $3.5 \%$ hypochloric acid, a morphological examination of the eggs was made.

\section{RESULTS}

Fecundity: The fertility of a female depends on her fecundity, i.e., on the number of eggs she deposits, and on the ability of these eggs to develop into adult flies. Table 1 summarizes observations on the number of eggs deposited by homozygous and by lethal-free heterozygous females. In agreement with the previous observations (MARINKovic 1967), it can be seen that the fecundity of the homozygotes is considerably more variable than that of the heterozygotes. Although a few individuals have produced no eggs during the six-day period of observation (only one culture from 354 analysed), none of the 118 homozygotes had zero fecundity. Two of the homozygotes approached this condition, producing fewer than $10 \%$ of the average eggs obtained from the heterozygotes (actually 3 and 19 eggs per female per six-day period); 23 homozygotes were semifecund, giving between 10 and $50 \%$ of eggs compared with the average heterozygote. The proportion of the semisterile (or semifecund) homozygotes was $21.2 \%$, compared with $13.7 \%$ obtained by Marinkovic (1967). The proportion of the chromosomes which can be regarded as quasinormal for fecundity is $78.8 \%$, which is not far from the proportion found previously - 86.3\%.

The average egg-laying capacity of a homozygote is significantly lower (161.5 \pm 5.7 eggs per six-day period) in comparison with random lethal-free heterozygotes $(207.5 \pm 5.9)$. This difference is about the same $\left(22 \%, t_{180}=5.229\right)$ as in our earlier data, when the fecundity of the females was analysed during a ten-day period $\left(19.2 \%, \mathrm{t}_{324}=5.754\right)$.

\section{TABLE 1}

Numbers of chromosomes (homozygotes), and of chromosomal combinations (heterozygotes), which produce different counts of eggs per female per six days of observation

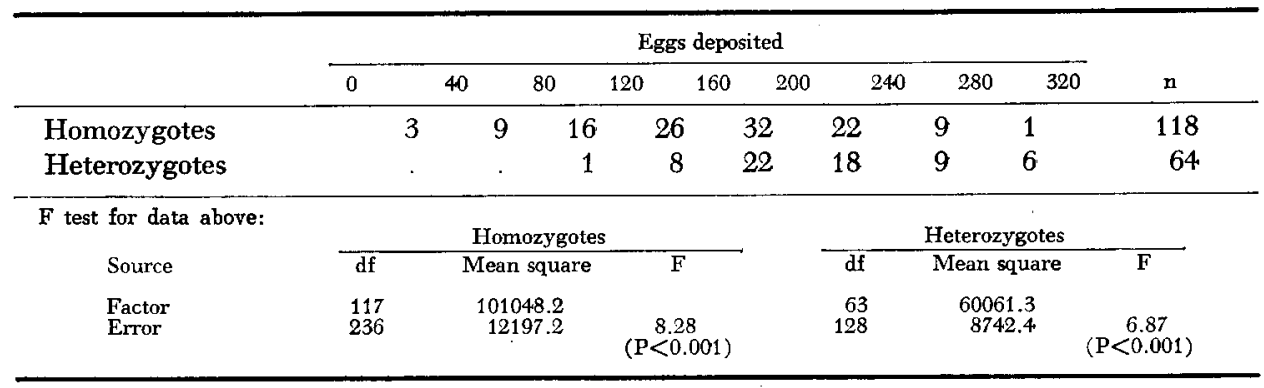


Egg-to-adult survival: It was of special interest to see if the homozygosity for wild-second chromosomes in the females, crossed to fertile males of other strains (Delta/Bare mutants have been used) will produce any effects on the survival of their eggs to the adult stage. Females heterozygous for wild-type chromosomes were raised as a control. The frequency distributions of the analysed chromosomes and chromosomal combinations according to the percentage of the survival of their eggs to the adult stage are shown in Table 2. Survival of the eggs laid by homozygous mothers is much lower than in the heterozygous control. Only $44.9 \pm 2.2 \%$ of the eggs laid by an average homozygote develop to the adult stage, while this percentage for their random heterozygous combinations is $69.9 \pm 1.8$. The difference between these two means is highly significant.

Four out of 118 homozygotes for the second chromosomes (3.9\%) did not produce any survivors from their eggs, despite the fact that the mothers had been inseminated and that motile spermatozoa were found in their sperm receptacles. In cultures containing more than 400 eggs, not a single larva was found; the sterility was due to failure of the eggs to complete embryogenesis.

In three other homozygous lines, where survival of eggs laid by homozygous females was extremely low (between 2.4 and $11.6 \%$ ), attempts have been made to determine when in embryogenesis mortality occurred. In $55 \%$ of the eggs, analysed 48 hours or later after the deposition, moving larvae were found within the envelopes of the chorion. In $11 \%$ of cases, zygotic mortality occurred at some earlier stage, but not earlier than before blastoderm formation. In $34 \%$ of the eggs, I was unable to determine whether any embryogenesis occurred; if these eggs had been inseminated, development must have stopped at some very early stage. In about 900 analysed eggs laid by 25 females homozygous for one of three chromosomes, all of the above mentioned conditions were observed, with mortality usually occurring at a particular stage of the development. In one of the analysed homozygotes, mortality occurred in $75 \%$ of the eggs after organogenesis was completed; in another, mortality happened in about $73 \%$ of the eggs at an earlier stage of development (before blastoderm formation, if insemination took place). In a third homozygote, the proportions of these two groups (mortality before or after the formation of the blastoderm) were approximately the same.

TABLE 2

Survival to the adult stage of the eggs laid by homozygous and heterozygous females

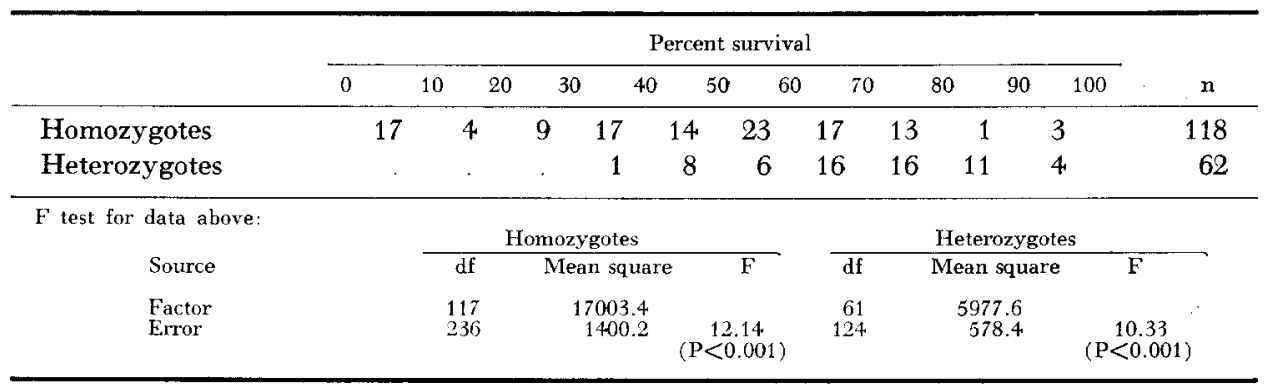


TABLE 3

Numbers of chromosomes (homozygotes), and of chromosomal combinations (heterozygotes), which produce different numbers of adult progeny per female per six days of oviposition

\begin{tabular}{lrrrrrrrrrrrr}
\hline & \multicolumn{10}{c}{ Number of progeny } & & \\
\cline { 2 - 8 } & \multicolumn{1}{c}{} & 30 & 60 & 90 & 120 & 150 & 180 & 210 & 240 & \multicolumn{1}{c}{$\mathbf{n}$} \\
\hline Homozygotes & 25 & 19 & 32 & 18 & 13 & 7 & 3 & 1 & 118 \\
Heterozygotes & & 1 & 3 & 11 & 19 & 19 & 7 & 2 & 62 \\
\hline
\end{tabular}

However, such variations have been found even among the eggs laid by the same female.

Fertility: The deleterious genes which affect the fecundity of the females homozygous for wild-type second chromosomes, and those which reduce the survival of their eggs, cause a drastic decrease in the number of progeny which these individuals produce. Table 3 shows the frequencies of the homozygotes and heterozygotes according to fertility, measured as the numbers of adult progeny per female per six days of egg laying (i.e. fecundity/female/ 6 days $\times$ percent survival). The average fertility of the homozygotes is $77.0 \pm 4.7$, in comparison with lethal free heterozygotes-143.6 \pm 4.8 . In other words, the number of the flies produced by a homozygous female is about $48 \%$ smaller than by an average heterozygous individual.

Table 3 shows that only 14 out of the 118 homozygotes had fertility greater than an average lethal-free heterozygote. Almost as many homozygotes (12 out of 118) were partially sterile, producing less than $10 \%$ of the progeny in comparison with a random heterozygous combination. Six homozygotes were sterile and produced, on the average, less than one individual per female in the next generation.

Table 4 summarizes the average values for the analysed components of fitness, including viability. Heterozygous combinations containing chromosomes with

\section{TABLE 4}

Viability (percent wild type), fecundity (eggs in six days), survival (percent survivors from eggs to adults) and fertility (the product of fecundity and survival) for the homozygotes and the heterozygous controls

\begin{tabular}{lcccc}
\hline & & \multicolumn{3}{c}{ Heterozygotes } \\
\cline { 3 - 5 } & Homozygotes & Total & QN/QN & Lethal/QN \\
\hline Viability & $28.4 \pm 0.5$ & $33.9 \pm 0.4$ & $33.9 \pm 0.5$ & $34.1 \pm 0.7$ \\
& $\mathrm{n}=118$ & $\mathrm{n}=87$ & $\mathrm{n}=64$ & $\mathrm{n}=23$ \\
Fecundity & $161.5 \pm 5.7$ & $210.1 \pm 4.9$ & $207.5 \pm 5.9$ & $217.5 \pm 8.8$ \\
& $\mathrm{n}=118$ & $\mathrm{n}=87$ & $\mathrm{n}=64$ & $\mathrm{n}=23$ \\
Survival & $44.9 \pm 2.2$ & $67.9 \pm 1.6$ & $69.9 \pm 1.8$ & $61.8 \pm 3.4$ \\
& $\mathrm{n}=118$ & $\mathrm{n}=82$ & $\mathrm{n}=62$ & $\mathrm{n}=20$ \\
Fertility & $\mathbf{7 7 . 0} \pm 4.7$ & $142.5 \pm 4.5$ & $143.6 \pm 4.8$ & $139.3 \pm 11.5$ \\
& $\mathrm{n}=118$ & $\mathrm{n}=82$ & $\mathrm{n}=62$ & $\mathbf{n}=20$ \\
& & & &
\end{tabular}


TABLE 5

Correlation coefficients for viability, fecundity and egg-to-adult survival in homozygotes and heterozygous combinations

\begin{tabular}{lcc}
\hline Viability and Fecundity & $\begin{array}{c}\text { Homozygotes } \\
(\mathrm{n}=118)\end{array}$ & $\begin{array}{c}\text { Heterozygotes } \\
(\mathbf{n}=62 \text { to 64) }\end{array}$ \\
\hline Viability and Survival & $\begin{array}{c}0.146 \\
(\mathrm{P}>0.1)\end{array}$ & 0.041 \\
0.195 & 0.007 \\
Fecundity and Survival & $\begin{array}{c}0<0.05) \\
0.304 \\
(\mathrm{P}<0.01)\end{array}$ & 0.041 \\
\hline
\end{tabular}

lethals and those with quasi-normal chromosomes are both shown. The average viability of the 118 homozygotes whose fecundity and egg-to-adult survival have been analysed is $28.4 \pm 0.5$. Including the lethals and extreme semilethals (i.e. cultures giving less than $10 \%$ wild-type flies), the average for homozygotes drops to $23.1 \pm 1.0(\mathrm{~N}=146$ chromosomes $)$. The proportion of the lethal and semilethal homozygotes is $22.6 \%$ (33 out of 146 analysed chromosomes). This value and the values for the homozygotes and heterozygous combinations, are within the range recorded for populations of $D$. pseudoobscura by Dobzhansky and Spassky (1953), Dobzhansky et al. (1963) and by other authors. It can be seen that the averages for viability and fecundity are slightly higher in lethal-carrying heterozygotes than in quasinormal combinations; however, the survival appears to be lower among the former than among the later. All these differences are not significant, and the numbers of progeny per female for the six-day period are approximately the same for both groups of heterozygotes $(143 \pm 5$ and $139 \pm 11)$. Hrraizumi and Crow (1960) found in D. melanogaster that lethal-carrying heterozygotes produce significantly fewer progeny in early stages of their life, in contrast with lethal-free combinations of chromosomes.

Correlation between viability, fecundity and egg-to-adult survival: Table 5 presents the correlation coefficients between the viability, fecundity and egg-toadult survival of the homozygotes and nonlethal heterozygotes. For the heterozygotes, no coefficient of correlation is significantly different from zero. For the homozygotes, a nonsignificant positive correlation is found between the viability and the fecundity $(P<0.1)$. This confirms the finding reported by Marinkovic (1967). However, the viability and the egg-laying capacity of the mothers are significantly positively correlated with the percentages of the survival of their eggs. Comparison between the viability of the females and the survival of their progeny gives a coefficient of correlation of $0.2(\mathrm{P}<0.05)$, and between their fecundity and the egg-to-adult survival an even stronger correlation of 0.3 $(\mathrm{P}<0.01)$.

Comparison of the 12 least viable homozygotes (less than $20 \%$ non-Bare flies in the cultures) and the 12 most viable ones (viability greater than $35.8 \%$ ) gave the following results for the fecundity and the egg-to-adult survival: 


$\begin{array}{lcc} & \text { Eggs/female/6 days } & \text { Egg-to-adult survival } \\ \text { Least viable homozygotes } & 154.5 \pm 23.9 & 32.3 \pm 7.6 \\ \text { Possible supervitals } & 174.2 \pm 12.6 & 53.2 \pm 5.7\end{array}$

A similar comparison between the 12 least fecund (less than 71 eggs/female/ 6 days) and the 12 most fecund (more than 236 eggs/female/ 6 days) homozygotes, gave the following results for viability and egg-to-adult survival:

$\begin{array}{lcc} & \begin{array}{c}\text { Percent of } \\ \text { non-Bare flies in cultures }\end{array} & \text { Egg-to-adult survival } \\ \text { Least fecund homozygotes } & 25.8 \pm 1.6 & 24.2 \pm 9.2 \\ \text { Most fecund homozygotes } & 28.9 \pm 1.8 & 58.9 \pm 4.8\end{array}$

The relationship between viability and fecundity is in principle the same as reported by Marinkovic (1967), i.e., an increase of fecundity among the supervitals, and a decrease of viability among the least fecund homozygotes. Egg-toadult survival is ostensibly decreased in both the least viable and the least fecund homozygotes, but it is markedly increased among the possible supervitals and in the most fecund homozygotes. The positive correlation between viability and fertility of the homozygotes, reported by SwEET and SPIEss (1962) and by TEMIN (1966) in D. melanogaster, is due to a very complex relationship between these components of fitness. The fecundity, egg-to-adult survival and viability of the homozygotes ordered according to their fertility, are presented in Table 6.

Among the six sterile homozygotes found in my material, the survival, fecundity and viability are as follows:

$\begin{array}{cc}\begin{array}{c}\text { Average progeny } \\ \text { per female per } 6 \text { days }\end{array} & \begin{array}{c}\text { Percent } \\ \text { egg-to-adult survival }\end{array} \\ 0.00 & 0.00 \\ 0.00 & 0.00 \\ 0.00 & 0.00 \\ 0.00 & 0.00 \\ 0.22 & 7.69 \\ 0.76 & 0.77\end{array}$

$\begin{array}{cc}\begin{array}{c}\text { Eggs per } \\ \text { female per } 6 \text { days }\end{array} & \text { Viability } \\ 45.1 & 31.4 \\ 19.1 & 33.1 \\ 151.1 & 26.9 \\ 182.1 & 32.0 \\ 2.9 & 16.0 \\ 65.9 & 25.5\end{array}$

Only in one case was viability low (it was a mild semilethal), but in three other sterile homozygotes it was even higher, compared with the average found in all

TABLE 6

Fecundity, egg-to-adult survival and viability of the homozygotes ordered according to their fertility

\begin{tabular}{ccccc}
\hline $\begin{array}{c}\text { Average } \\
\begin{array}{c}\text { progeny per } \\
\text { female per 6 days }\end{array}\end{array}$ & $\begin{array}{c}\text { Number of } \\
\text { homozygotes }\end{array}$ & Mean fecundity & $\begin{array}{c}\text { Mean percent } \\
\text { survival of laid eggs }\end{array}$ & Mean viability \\
\hline $0-40$ & 28 & $105.6 \pm 11.6$ & $13.6 \pm 2.1$ & $25.9 \pm 1.0$ \\
$41-80$ & 36 & $154.1 \pm 7.8$ & $43.4 \pm 2.9$ & $29.1 \pm 1.0$ \\
$81-120$ & 30 & $170.3 \pm 6.0$ & $58.8 \pm 1.9$ & $29.3 \pm 0.8$ \\
$121-160$ & 16 & $214.1 \pm 5.8$ & $64.4 \pm 3.5$ & $29.4 \pm 1.5$ \\
$161-200$ & 6 & $239.1 \pm 11.6$ & $75.2 \pm 4.2$ & $27.5 \pm 3.2$ \\
$201-240$ & 2 & $296.5 \pm 18.8$ & $71.4 \pm 1.9$ & $33.6 \pm 2.0$ \\
\hline
\end{tabular}


homozygotes. Complete sterility is not necessarily associated with insufficient viability. Complete sterility among the homozygotes in my material was produced mostly by failure of the eggs to develop to the adult stage, and in one case also by a decrease in the fecundity of the females. It should be noticed, however, that in four out of six sterile homozygotes the fecundity was also considerably reduced.

\section{DISCUSSION}

Dobzhansky and Spassky (1953) tested for fertility females and males of D. pseudoobscura homozygous for $2 \mathrm{nd}$, $3 \mathrm{rd}$, or 4th chromosomes found in natural populations. Among the homozygotes of quasinormal viability, the following percentages of chromosomes induced complete sterility of the females: $10.6 \%$ for second, $13.6 \%$ for third, and $4.3 \%$ for the fourth chromosomes. SweEt and SpIEss (1962) found in D. melanogaster $7.9 \%$ of the homozygotes for the second and $7.8 \%$ for the third chromosomes completely sterile as females; a comparable figure obtained for second chromosomes by TEMIN (1966) is $4.2 \%$. In the present study, only $5.1 \%$ (6 out of 118 ) of second chromosomes of D. pseudoobscura, giving quasinormal or mild semilethal viabilities, proved almost completely sterile, yielding fewer than one individual in the progeny after a six-day period of egg laying. In five of these six homozygotes, the sterility was due to very low survival of the eggs deposited by the homozygous females, even though they were inseminated by fully fertile males and had motile sperm in their seminal receptacles. The defect was evidently due to the homozygous females producing abnormal eggs, that resulted in giving the inviable progenies. Embryogenesis in these eggs was in some cases completed, but the larvae failed to hatch. The sixth homozygote had an extremely low fecundity as well as low survival of the progeny.

TEMrN (1966) found a bimodal distribution of fertility among homozygotes for second chromosomes in D. melanogaster. Her data consisted however of numbers of cultures producing any progeny, or no progeny, among individuals homozygous for a given second chromosome. The observations reported in the present article show that, at least in D. pseudoobscura, the bimodality is due to the distribution of the egg-to-adult survival, while the egg-laying capacity (fecundity) shows a unimodal distribution. TEMIN's technique, as she points out, is likely to underestimate the loss of fertility caused by homozygosis for second chromosomes taken from nature; she found that in D. melanogaster about $90 \%$ of the average fertility is retained in the homozygotes. My data for $D$. pseudoobscura show that only about $54 \%$ of the average fertility is retained. The loss of fertility is compounded of an approximate $22 \%$ loss of fecundity and a $36 \%$ decrease of survival of progeny. The loss of fitness through diminished fertility may, thus, be greater than that arising from diminished viability of the homozygotes $(32 \%)$.

SweEt and SpIEss (1962) and Temin (1966) found a positive but nonsignificant correlation between the fertility and viability of the homozygotes. My data (Marinkovic 1967; Table 5 above) show a positive correlation between the 
viability of homozygous females and egg-to-adult survival of their progeny. Eleven homozygotes which gave less than 10\% egg-to-adult survivors (complete steriles are not induced) showed also a considerably decreased fecundity (142.4 \pm 14.0 eggs per female per six days) and viability (24.1 $\pm 3.4 \%)$.

Dobzhansky et al. (1942), Sweet and Spiess (1962) and Temin (1966) found an association between low viability and complete sterility of homozygous females. Pavan et al. (1951) indicated in $D$. willistoni that sterility is not connected with low viability, or vice versa (lethals and semilethals were excluded). SweEt and SpIEss (1962) also reported sterility factors in females of $D$. melanogaster causing no decrease in viability. In the present experiment, no consistent association was found between complete sterility of females homozygous for wild second chromosomes, and their viabilities. Among the sterile homozygotes only one was a mild semilethal, but three others had a greater than average viability. Sterility was caused mostly by failure of the eggs laid to survive, but sterile females had also significantly decreased fecundity ( 78 eggs per female per six days).

The author is grateful to Professon Th. Dobzhansky for numerous suggestions throughout the course of these experiments and for his helpful comments on the preparation of the manuscript. Warmest thanks are due to Mr. R. C. Richmond for his assistance in the statistical analysis and programming of the computations of the results on the $160-\mathrm{G}$ computer. The dissection technique and the technique for the analysis of the embryogenesis were shown to me by Drs. L. Ehrman and O. Kitagawa. The author also wishes to express his thanks to Drs. F. J. Ayala and W. W. Anderson, as well as the colleagues mentioned above, for many useful discussions.

\section{SUMMARY}

A total of 118 chromosomes from natural populations were studied for their effects on the fecundity of homozygous females and on egg-to-adult survival of their progeny; 64 heterozygous combinations of second chromosomes from the same populations were tested for comparison. The mean number of eggs deposited by a homozygous female during six days at the beginning of her egg laying period is $161.5 \pm 5.7$, and only $44.9 \pm 2.2 \%$ of them survive to the adult stage. The corresponding figures for heterozygous females are $207.5 \pm 5.9$ eggs, and $69.9 \pm 1.8 \%$ survival. Homozygotes are $46.4 \%$ less fertile than heterozygotes. $5.1 \%$ of the homozygotes were completely sterile, owing mainly to inability to complete embryogenesis and to a less extent to a lowered fecundity. The survival of eggs of homozygous females is positively correlated with the viability and fecundity of their mothers. The average number of progeny per heterozygous female was the same in heterozygotes containing lethals and in lethal-free combinations of wild second chromosomes.

\section{LITERATURE CITED}

Dobzhansky, Th., A. M. Holz, and B. Spassky, 1942 Genetics of natural populations. VIII. Concealed variability in the second and the fourth chromosomes of Drosophila pseudoobscura and its bearing on the problem of heterosis. Genetics $27: 463-490$. 
Dobzhansky, Th., A. S. Hunter, O. Pavlovsky, B. Spassky, and B. Wallace, 1963 Genetics of natural populations. XXXI. Genetics of an isolated marginal population of Drosophila pseudoobscura. Genetics 48: 91-103.

Dobzhansky, Th., and B. Spassky, 1953 Genetics of natural populations XXI. Concealed variability in two sympatric species of Drosophila. Genetics 38 : 471-484.

Hiraizumi, Y., and J. F. Crow, 1960 Heterozygous effects on viability, fertility, rate of development, and longevity of Drosophila chromosomes that are lethal when homozygous. Genetics 45: 1071-1083.

Marinkovic, D., 1967 Genetic loads affecting fecundity in natural populations of Drosophila pseudoobscura. Genetics 56: 61-71.

Pavan, C., A. R. Cordeiro, Th. Dobzhansky, N. Dobzhansky, C. Malogolowkin, B. Spassky, and M. WeDEL, 1951 Concealed genic variability in Brazilian populations of Drosophila willistoni. Genetics 36: 13-30.

Sweer, E. E., and E. B. Spiess, 1962 Frequency of sterility in a laboratory population of Drosophila melanogaster. Genetics 47: 1519-1534.

Temin, R. G., 1966 Homozygous viability and fertility loads in Drosophila melanogaster. Genetics 53: 27-46. 